\title{
Thermal transport in one-dimensional van der Waals heterostructures: Effects of coating layers on the thermal conductivity of carbon nanotubes
}

\author{
Penghua Ying, Jin Zhang*, Yao Du, Zheng Zhong* \\ School of Science, Harbin Institute of Technology, Shenzhen 518055, PR China \\ *Corresponding author. zhongzheng@hit.edu.cn (Z.Z.) jinzhang@hit.edu.cn (J.Z.)
}

\begin{abstract}
:
In this paper, we conduct a comprehensive investigation on the thermal transport in one-dimensional (1D) van der Waals (vdW) heterostructures by using nonequilibrium molecular dynamics simulations. It is found that the boron nitride nanotube (BNNT) coating can increase the thermal conductance of inner carbon nanotube (CNT) base by $36 \%$, while the molybdenum disulfide nanotube (MSNT) coating can reduce the thermal conductance by $47 \%$. The different effects of BNNT and MSNT coatings on the thermal transport behaviors of $1 \mathrm{D} \mathrm{vdW}$ heterostructures are explained by the competition mechanism between improved heat flux and increased temperature gradient in 1D vdW heterostructures. By taking CNT@BNNT@MSNT as an example, thermal transport in $1 \mathrm{D} \mathrm{vdW}$ heterostructures containing three layers is also investigated. It is found that the coaxial BNNT-MSNT coating can significantly reduce the thermal conductance of inner CNT base by $61 \%$, which is even larger than that of an individual MSNT coating. This unexpected reduction in thermal conductance of CNT@BNNT@MSNT can be explained by the suppression of heat flux arising from the possible compression effect, since BNNT-MSNT coating in CNT@BNNT@MSNT can more significantly suppress the vibration of inner CNT when compared to the individual MSNT coating in CNT@MSNT. In addition to the in-plane thermal transport, the interfacial thermal conductance between inner and outer nanotubes in $1 \mathrm{D} \mathrm{vdW}$ heterostructures is also examined to provide a quantitative understanding of the thermal transport behaviors of1D vdW heterostructures. This work is expected to provide molecular insights into tailoring the heat transport in carbon base $1 \mathrm{D} v \mathrm{vW}$ heterostructures and thus facilitate their broader applications as thermal interface materials.
\end{abstract}

\section{Introduction}

The two-dimensional (2D) heterostructures have attracted much attention recently by virtue of merging different $2 \mathrm{D}$ crystals to enable novel physical properties and functionalize applications, ${ }^{1}$ such as band-structure engineering, ${ }^{2}$ ultrafast charger 
transfer, ${ }^{3}$ and vertical field-effect transistor. ${ }^{4}$ According to the direction of combination, 2D heterostructures can be divided into vdW heterostructures and in-plane heterostructures. The vdW heterostructures are synthesized by stacking different 2D crystals with weak vdW interactions in the out-of-plane direction. As for their in-plane counterparts, different coplanar 2D crystals are linked via covalent bonds. The development of 2D vdW heterostructures have greatly expanded the 2D material family. Inspired by the fact that a single-walled carbon nanotube (CNT) can be treated as a seamless cylinder rolled from graphene, it is expected that the corresponding 1D tubular vdW heterostructures could be obtained by rolling up 2D vdW heterostructures. However, compared with planar 2D sheets, 1D nanotubes are much more difficult to be synthesized in experiments, since their structures are topologically protected from being stack-nested. ${ }^{5}$ Hence, many efforts have been devoted to make progress in synthesizing $1 \mathrm{D}$ vdW heterostructures. Very recently, Xiang et al. ${ }^{6}$ demonstrated the experimental discovery and controlled fabrication of 1D tubular vdW heterostructures with single crystals, which consist of three different shell components: an inner CNT, a middle hexagonal boron nitride nanotube (BNNT), and an outer molybdenum disulfide nanotube (MSNT). In addition to this triple-walled nanotube heterostructure, doublewalled nanotube heterostructures such as CNT@BNNT and CNT@MSNT were also obtained in their experiment. The obtained 1D vdW heterostructures were found to exhibit unique optoelectronic properties. ${ }^{7}$

Due to their superior thermal conductivity and outstanding mechanical stability, these $1 \mathrm{D} v \mathrm{vdW}$ heterostructures can serve as promising thermal interface materials in solving the problem of heat dissipation in modern electronic devices. ${ }^{8}$ However, the thermal property of 1D heterostructures is still almost unexplored despite that the thermal transport behaviors of their pristine counterparts such as $\mathrm{CNT},{ }^{9,10} \mathrm{BNNT},{ }^{11}$ and MSNT, ${ }^{12,13}$ have been well investigated. To our best knowledge, among different 1D vdW heterostructures, only the thermal conductivity of CNT@BNNT has just been investigated. ${ }^{14}$ Based on the experimental measurement and molecular dynamics (MD) simulations, Jing et al. ${ }^{14}$ reported the enhancement in the thermal conductivity of coaxialCNT@BNNT arrays when compared to the isolated CNT arrays. To data, however, the thermal transport property in other $1 \mathrm{D}$ vdW heterostructures such as CNT@MSNT and CNT@BNNT@MSNT which have been successfully synthesized by Xiang et al. ${ }^{6}$ remains unknown. Moreover, there is no related report on the interfacial 
thermal conductance inside $1 \mathrm{D}$ vdW heterostructures, in addition to their counterparts of $2 \mathrm{D}$ heterostructures. ${ }^{15,16}$ However, understanding interfacial thermal transport in 1D vdW heterostructures, especially at the edge between long inner CNT and short outer coating nanotubes observed in the experiments, ${ }^{6}$ is crucial for their applications as nanoelectronics devices, ${ }^{17}$ since heat can not only transport along axial direction, but also conduct through the interface of nanotubes.

To this end, taking $(40,40) \mathrm{CNT}$ as the base structure, 1D vdW double-walled nanotubes(CNT@CNT, CNT@BNNT,CNT@MSNT) and triple-walled nanotubes (CNT@BNNT@MSNT) heterostructures were constructed to investigate their enhancement/hindering effect by non-equilibrium molecular dynamics (NEMD) method. We also calculated the heat flux, temperature distributions together with vibrational density of states (VDOS) to reveal the mechanism for their corresponding enhancement/hindering effect. It was found that both CNT@CNT and CNT@BNNT shows an enhancement effect on thermal transport in base CNT, while CNT@MSNT and CNT@BNNT@MSNT exhibits the opposite hindering effect. As an initial attempt, we also calculated the interfacial thermal conductance between nanotubes in doublewalled heterostructures, which is much weaker than their counterpart along axial direction.

\section{Models and methods}

Models. As plotted in Figure 1, CNT, BNNT and MSNT are considered in our study. According to the number of layers of nanotubes, the nanotube materials can be divided into single-walled, double-walled, and triple-walled nanotubes. It should be noted that our study only focuses on armchair nanotubes and ignores other chiral nanotubes. Specifically, an armchair CNT (BNNT or MSNT) with the lattice vector of $\mathbf{r}=m \boldsymbol{a}_{1}+m \boldsymbol{a}_{2}\left(\boldsymbol{a}_{1}=\boldsymbol{a}_{2}\right.$ are lattice constants of the primitive cell $)$ can be noted as $m m \mathrm{CNT}$ (BNNT or MSNT). In the present study, 4040CNT is selected as the base nanotube, which is coated by 4545CNT, 4545BNNT and 3838MSNT (see Figure 1(a)). In other words, double-walled nanotubes including 4040CNT@4545CNT, 4040CNT@4545BNNT and4040CNT@3838MSNT, and the triple-walled nanotube 4040CNT@4545BNNT@4242MSNT as shown in Figure 1(c) are considered in the present study. For convenience, these nanotube structures are referred to as CNT@CNT, CNT@BNNT, CNT@MSNT and CNT@BNNT@MSNT, respectively. The specific atomic structures of these investigated coaxial heterostructures are plotted in Figure 
1(a)-(c).

To simplify the modelling of the coaxialCNT@BNNT structures, the bond lengths of CNT and BNNT are both set as $1.43 \AA$, which is an intermediate value between the intrinsic values $1.42 \AA$ and $1.45 \AA$ for CNT and BNNT, respectively. The bond length of MSNT, i.e., the distance between adjacent Mo and S atoms is set as $2.42 \AA$. Thus, CNT and MSNT can be perfectly matched as a CNT@MSNT supercell when 4 MSNT unit cells are on top of 5 CNT unit cells as shown in Figure 1(d), which is similar to the structure of the supercell of graphene/MoS 2 heterostructures. ${ }^{18,} 19$ The inter-wall distance between the inner CNT layer and outer CNT layer (or BNNT layer) is set as $3.4 \AA$, while the inter-wall distance between the CNT (BNNT) layer and the adjacent MSNT layer is set as $3.6 \AA$. These values of inter-wall distance are very close to the results predicted from previous first-principle calculations for $2 \mathrm{D}$ heterostructures. ${ }^{20-22}$

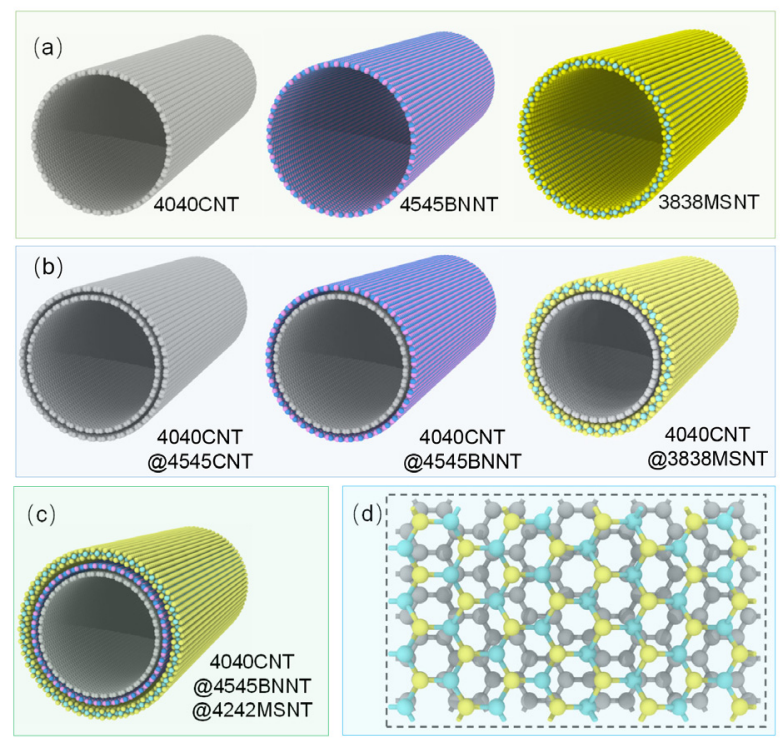

Figure 1. (a) Single-walled nanotubes: 4040CNT, 4545BNNT, and 3838MSNT; (b) double-walled nanotubes: 4040CNT@4545CNT, 4040CNT@4545BNNT, and 4040CNT@3838MSNT; (c) triplewalled nanotubes: 4040CNT@4545BNNT@4242MSNT; (d) one supercell of CNT@MSNT heterostructure, the lattice constant of MSNT and CNT along axial direction are 3.096 and $2.477 \AA$ respectively.

Molecular dynamics simulations. The molecular dynamics simulation conducted in the present study is implemented by using the publicly available simulation code LAMMPS $^{23}$, in which the standard Newton equations of motion were integrated in time using the velocity Verlet algorithm. The time step was set as 1.0 fs. All initial structures 
were first adopted to perform energy minimization. Then, the structures were further relaxed at $300 \mathrm{~K}$ and $0 \mathrm{GPa}$ along the axial direction for $100 \mathrm{ps}$ using the Langevin thermostat ${ }^{24}$ to obtain the equilibrium state. The interactions among $\mathrm{C}, \mathrm{B}$ and $\mathrm{N}$ atoms were described by the Tersoff potential, ${ }^{25,26}$ while the interaction between Mo and S atoms in MSNTs was described by the REBO potential, since the previous $\operatorname{study}^{27}$ suggested that the REBO potential ${ }^{28,29}$ can give a more accurate prediction of thermal transport properties compared to the Stillinger-Weber potential. ${ }^{30,31}$

The weak vdW interactions between adjacent nanotubes are modelled by 12-6 Lennard-Jones (LJ) potential with the form $V\left(r_{i j}\right)=4 \varepsilon\left[\left(\sigma / r_{i j}\right)^{12}-\left(\sigma / r_{i j}\right)^{6}\right]$, where $\varepsilon, \sigma$, and $r_{i j}$, respectively, are depth of the potential well, finite distance at which the inter-particle potential is zero, and the distance between atoms $i$ and $j$. The parameters $\varepsilon$ and $\sigma$ are set to be $\varepsilon=2.620 \mathrm{meV}$ and $\sigma=0.3440 \mathrm{~nm}$ among $\mathrm{C}$ atoms, $\varepsilon=4.116 \mathrm{meV}$ and $\sigma=0.3453$ $\mathrm{nm}$ among $\mathrm{B}$ atoms, $\varepsilon=0.586 \mathrm{meV}$ and $\sigma=0.4200 \mathrm{~nm}$ among Mo atoms, and $\varepsilon=$ $13.860 \mathrm{meV}$ and $\sigma=0.3130 \mathrm{~nm}$ among $\mathrm{S}$ atoms. ${ }^{28,32-34}$ The arithmetic mix rule is employed to model the LJ potential between different elements. Meanwhile, the cutoff in the LJ potential is set as $1.0 \mathrm{~nm}$. The corresponding binding energy of one CNT@MSNT heterostructure supercell as plotted in Figure 1(d) extracted from the LJ potential is $-20.4 \mathrm{meV}$, which is in good agreement with the result around $-21.0 \mathrm{meV}$ of graphene/ $\mathrm{MoS}_{2}$ heterostructures previously obtained from first-principle calculations. ${ }^{35}$ 

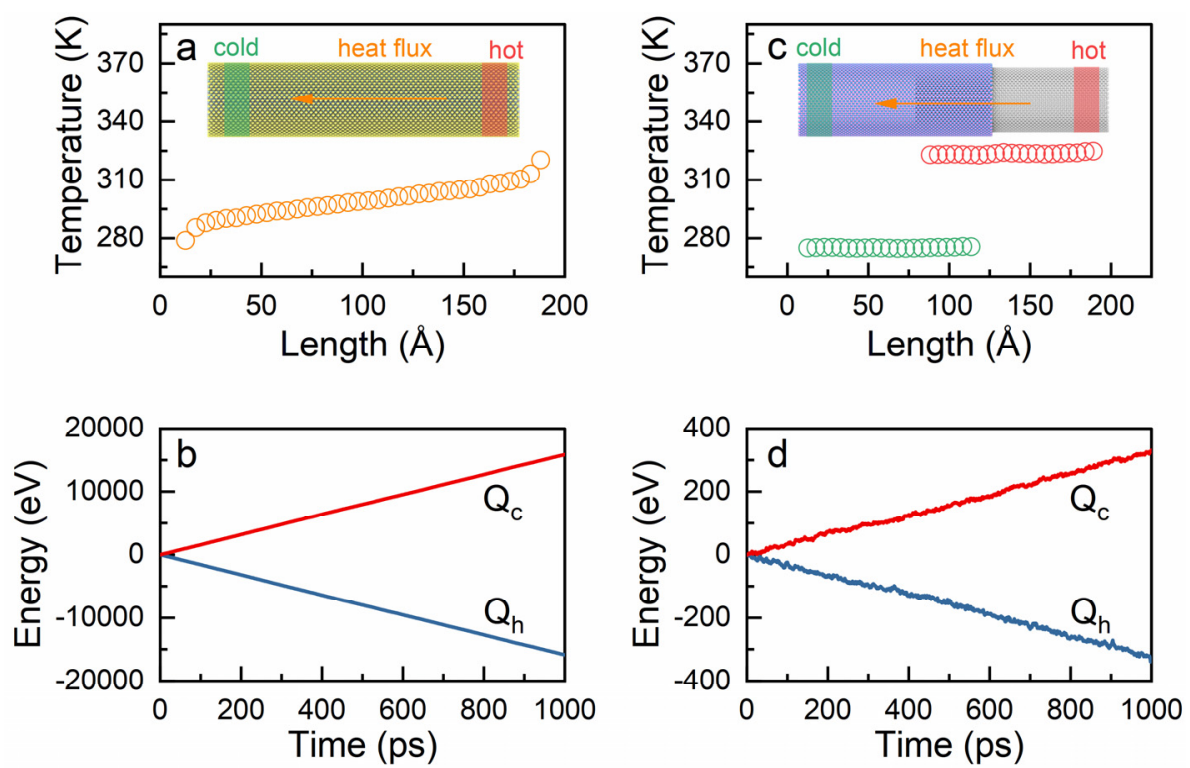

Figure 2. Schematic model setup for NEMD simulation: (a) the temperature profile and (b) accumulated energy changes of clod and hot region of 4040CNT@3838MSNT along axial direction during in-plane thermal conductivity calculation at $300 \mathrm{~K}$. (c) the temperature profile and (d) accumulated energy changes of clod and hot region of 4040CNT@4545BNNT along axial direction during interfacial thermal conductance calculation at $300 \mathrm{~K}$. The simulation domains are respectively plotted in the insects of (a) and (b).

Thermal conductivity calculations. The classical non-equilibrium MD method was employed in the calculations of thermal conductivity. Specifically, the thermal conductivities $k$ of investigated nanotubes was extracted with the aid of the following Fourier's law of heat conduction:

$$
k=-\frac{J}{A \nabla T} .
$$

Here $J$ denotes the heat flux along the axial direction of nanotubes. $\nabla T=d T / d x$ is the temperature gradient along the axial direction with $T$ and $x$ being the temperature and the axial coordinate, respectively. $A$ is the cross-sectional area of the nanotube, which can be approximated as $A=\pi\left[\left(R_{\text {out }}-r_{\text {out }}\right)^{2}-\left(R_{\text {in }}-r_{\text {in }}\right)^{2}\right]$. Here, $R_{\text {in }}$ and $R_{\text {out }}$ are, respectively, the radii of inner and outer nanotubes, while $r_{\text {in }}$ and $r_{\text {out }}$ are, respectively, the thicknesses of inner and outer nanotubes, which are assumed to equal to their vdW diameters. In this study, the thicknesses of CNT, BNNT and MSNT are assumed to be $0.344 \mathrm{~nm}$, $0.344 \mathrm{~nm}$ and $0.672 \mathrm{~nm}$, respectively, which are the same the thickness of their twodimensional materials counterparts. ${ }^{19}$ The thermal conductance $\sigma(\sigma=-J / \nabla T)$ of nanotubes was also obtained. In calculating $\sigma$, the cross-section area $A$ was fixed as 1 
$\AA^{2}$ to exclude the influence of cross-sectional area.

As shown in Figure 2(a), to perform non-equilibrium MD simulations, the whole nanotube was divided into five regions along the axial direction, which include two fixed regions at the two ends, the adjacent cold and hot regions (i.e., heat sink and heat sources), and the middle heat flux region. The lengths of fixed region and cold (or hot) region were set as $5 \AA$ and $10 \AA$, respectively. The temperatures of the cold and hot regions were set as $275 \mathrm{~K}$ and $325 \mathrm{~K}$, respectively. We divided the simulation box into 40 slabs along the axial direction to record the time-average temperature of each slab for generating the temperature gradient. The heat transport simulation lasts for $1500 \mathrm{ps}$. Specifically, the stable temperature distribution was achieved within the first $500 \mathrm{ps,}$ while the temperature gradient was obtained by averaging over the last 1000 ps MD simulations, which is graphically shown in Figure 2(b) for CNT@MSNT with a length of $20 \mathrm{~nm}$. Meanwhile, as shown in Figure 2(c), the corresponding heat flux $J$ was recorded by the averaging the input power of cold region and the output power of hot region through $J=\left(Q_{h}+Q_{c}\right) / 2 \Delta t$, where $Q_{c}$ and $Q_{h}$ are, respectively, the accumulated energy in cold and hot regions (i.e., heat sink and heat source regions) during the running time $\Delta t$.

Interfacial thermal conductance calculations. As shown in Figure 2(c) and (d), we adopted the similar NEMD method to calculate the interfacial thermal conductance. However, in the present calculation of interfacial thermal conductance, an artificial interface was created to make the heat flux $J$ pass through the overlapping interface area $A$ of the inner and outer nanotubes, which results in a significant temperature difference $d T$. Thus, the interfacial thermal conductance $G$ can be calculated from

$$
G=\frac{J}{A d T} \text {. }
$$

During the simulation, the whole structure was divided into five regions along the axial direction (see Figure 2(c)), which is exactly like the treatment we conducted in the above thermal conductivity calculation. Here, the hot region with a length of $5 \AA$ locates at the inner nanotube, while the cold region with the same length locates at the outer nanotube. The overlap length between the inner and outer nanotubes was set as 4 $\mathrm{nm}$. After performing $500 \mathrm{ps}$ relaxation simulations to achieve the steady heat flux transport, we calculated the temperature difference $d T$ between inner and outer nanotubes by averaging the temperatures of the overlap region in the subsequent 1000 
ps simulations.

Calculations of vibrational density of states (VDOS). The VDOS were studied to understand the underlying mechanisms of phonon transport, which can be calculated from the Fourier transform of the velocity autocorrection function ${ }^{36}$ :

$$
\operatorname{VDOS}(v)=\int \gamma(t) \exp (-2 \pi i v t) d t
$$

where $v$ denotes the frequency, $i$ is the imaginary unit. $\gamma$ denotes the velocity autocorrelation function obtained from the equation ${ }^{37} \gamma(t)=$ $\left\langle\sum_{i} v_{i}(0) \cdot v_{i}(t)\right\rangle /\left\langle\sum_{i} v_{i}(0) \cdot v_{i}(0)\right\rangle$ with $v_{i}(0)$ and $v_{i}(t)$ being the velocities of the $i$ th atom at time $t$ and at the initial state, respectively.

\section{Results and discussion}

\subsection{Thermal transport in double-walled nanotube heterostructures}

Table 1 . Thermal conductivity of various $20 \mathrm{~nm}$ NTs extracted from MD simulation.

\begin{tabular}{|c|c|c|c|c|c|c|}
\hline \multicolumn{2}{|r|}{ NTs } & $R_{\text {in }}(\AA)$ & $R_{\text {out }}(\AA)$ & $A\left(\AA^{2}\right)$ & $k(\mathrm{~W} / \mathrm{mK})$ & $\sigma\left(\times 10^{5} \mathrm{~W} / \mathrm{mK}\right)$ \\
\hline \multirow{4}{*}{ SWNTs } & 4040CNT & 27.31 & 1 & 590.28 & 630.75 & 3.723 \\
\hline & 4545CNT & 30.72 & 1 & 663.99 & 619.32 & 4.112 \\
\hline & 4545BNNT & 30.72 & 1 & 663.99 & 278.85 & 1.857 \\
\hline & 3838MSNT & 32.43 & 1 & 1369.37 & 12.82 & 0.176 \\
\hline \multirow{3}{*}{ DWNTs } & 4040CNT@4545CNT & 27.31 & 30.72 & 1248.80 & 651.41 & 8.135 \\
\hline & 4040CNT@4545BNNT & 27.31 & 30.72 & 1248.80 & 406.48 & 5.078 \\
\hline & 4040CNT@3838MSNT & 27.31 & 32.43 & 1966.88 & 99.67 & 1.960 \\
\hline
\end{tabular}

The thermal conductivity and conductance of various single-walled and doublewalled nanotubes with the same length of $20 \mathrm{~nm}$ are listed in Table 1. As for the singlewalled nanotubes considered here, it is found that the CNT has the highest thermal conductivity, which is $630.75 \mathrm{~W} / \mathrm{mK}$ for $4040 \mathrm{CNT}$ and $619.32 \mathrm{~W} / \mathrm{mK}$ for $4545 \mathrm{CNT}$, while the MSNT has the lowest thermal conductivity of $12.82 \mathrm{~W} / \mathrm{mK}$. The BNNT has a moderate thermal conductivity of $278.85 \mathrm{~W} / \mathrm{mK}$. The values of these three singlewalled nanotubes are in good agreement with the previous studies on the basis of NEMD methods. ${ }^{10,12,13}$ As for the double-walled nanotubes, the thermal conductivities ofCNT@CNT (i.e., double-walled CNT ),CNT@BNNT, and CNT@MSNT extracted from the present study are $651.41 \mathrm{~W} / \mathrm{mK}, 406.48 \mathrm{~W} / \mathrm{mK}$, and $99.67 \mathrm{~W} / \mathrm{mK}$, respectively. It is noted that the thermal conductivity of double-walled CNT is identical to the value of its single-walled counterpart, while the thermal conductivity of CNT@BNNT (or CNT@MSNT) is between the values of their component CNT and BNNT (or MSNT) 
layers.

In the above discussion the thickness of single-walled nanotubes is assumed to equal to the vdW diameter, but many other values also have been suggested for the equivalent thickness of single-walled nanotubes. ${ }^{38-40}$ Thus, to surmount the hurdle in identifying the equivalent thickness of the one atom or several atoms thick nanotube structures, the thermal conductance $\sigma$ defined above instead of the thermal conductivity $k$ is calculated here to eliminate the effect of thickness and thus the cross-sectional area. Moreover, to more clearly measure the contribution of the outer coating nanotube layer to the overall thermal conductance of $1 \mathrm{D}$ vdW heterostructures, we introduce the following parameter $\alpha$ :

$$
\alpha=\frac{\sigma_{\text {het }} \sigma_{\text {in }}}{\sigma_{\text {in }}},
$$

where $\sigma_{h e t}$ and $\sigma_{\text {int }}$ are the thermal conductance of $1 \mathrm{D} \mathrm{vdW}$ heterostructures and the inner $4040 \mathrm{CNT}$, respectively. If $\alpha>0$, the outer coating nanotube layer can improve the thermal conductance of the structure, otherwise the coating nanotube layer has the opposite effect. As shown in Figure 3, the values of $\alpha$ are 1.34, 0.36, and -0.47 for CNT@CNT,CNT@BNNT, andCNT@MSNT, respectively. This result indicates that both CNT and BNNT coatings can improve the thermal transport property of $1 \mathrm{D} v \mathrm{vdW}$ heterostructures, while the MSNT coating has an opposite effect, which can reduce the thermal transport property. Moreover, the thermal conductance of CNT@CNT is found to exactly equal to sum of the values of the inner CNT base and the outer CNT coating, which is thus twice as the value of the single-walled CNT. The value of CNT@BNNT is close to but slightly lower than the sum of the values of the inner CNT and the outer BNNT, which is thus still significantly higher than the value of the single-walled CNT. The different enhancement effects of CNT and BNNT coatings on the thermal conductivity of the single-walled CNT can be understood by the fact that the CNT has a thermal conductivity much higher than that of BNNT. In addition, the mass of nitrogen and boron atoms is different from that of the carbon atom. This difference can introduce an acoustic mismatch between the inner CNT and outer BNNT in CNT@BNNT, which can be regarded as another factor responsible for the above result that the thermal conductance of CNT@BNNT is slightly lower than the sum of the values of its inner CNT and outer BNNT components. The enhanced thermal conductance observed in CNT@CNT and CNT@BNNT is in accordance with our expectation, since an outer 
nanotube coating layer can supply an additional heat conducting path. However, different to CNT@CNT and CNT@BNNT, a reduced thermal conductance is found in CNT@MSNT, which is only one half of the value of the single-walled CNT. This reduction in the thermal conductance of CNT@MSNT is out of our expectation,which will be explained in details latter.

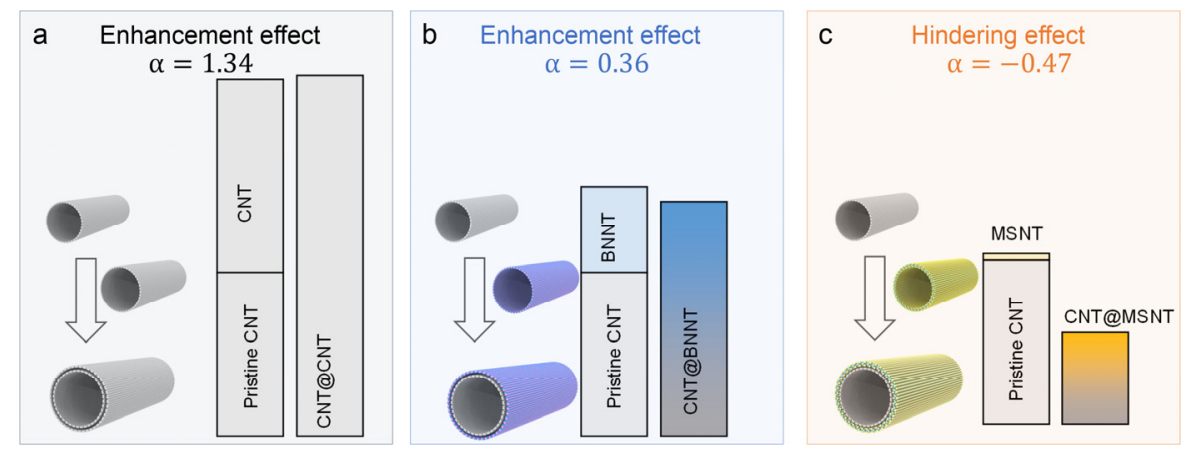

Figure 3. The effect of encapsulating different nanotubes including (a) 4545CNT, (b) 4545BNNT, and (c) $3838 \mathrm{MSNT}$ on the thermal conductivity of pristine 4040CNT. The height of each rectangle denotes the corresponding thermal conductance.

To explain the different effects of different nanotube coatings on the overall thermal conductance of corresponding $1 \mathrm{D} \mathrm{vdW}$ heterostructures, we show their temperature distribution in Figure 4(a)-(c) and the cumulative energy change in Figure 4(d)-(f), respectively. The results of the corresponding inner and outer nanotube components in 1D vdW heterostructures are also shown for the sake of comparison. A dramatic temperature drop near the heat source and the sink region is found in all calculated structures, which can be attributed to the unavoidable intensive phonon scattering. Under this circumstance, only the linear temperature distribution region ranging from $30 \AA$ to $170 \AA$ was considered in calculating the temperature gradient. The cumulative energy changes in the heat source and sink region are identical to each other, which increase linearly with respect to the growing simulation time. 

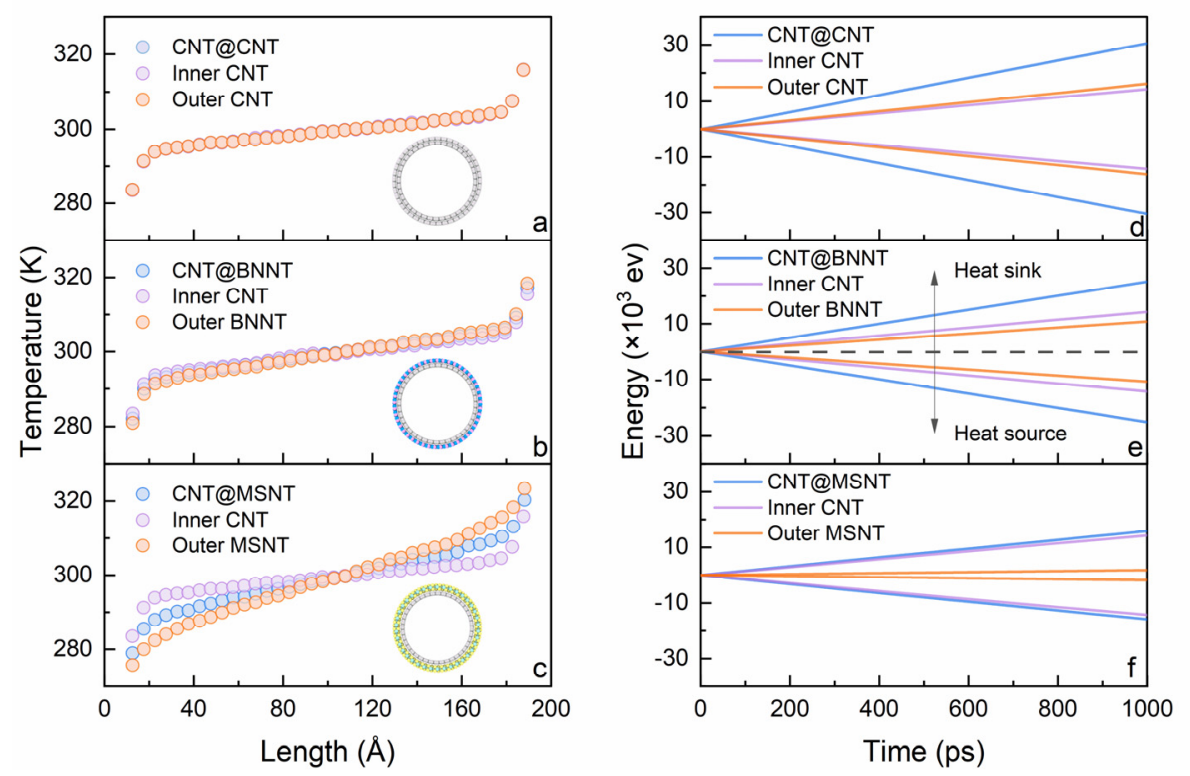

Figure 4. The temperature profiles of double-walled nanotubes including (a) 4040CNT@4545CNT, (b) 4040CNT@4545BNNT, and (c)4040CNT@3838MSNT along axial direction during NEMD simulation at $300 \mathrm{~K}$. For each heterostructure, the temperature distribution of the double-walled nanotube, inner nanotube, and outer nanotube are respectively calculated for sake of comparison. The accumulated energy changes of the heat sink and source in (d) 4040CNT@4545CNT, (e) 4040CNT@4545BNNT, and (f) 4040CNT@3838MSNT during NEMD simulation at 300K. Noted that in (b)-(d), the energy changes of isolated inner and outer nanotubes are calculated instead of their counterpart in heterostructures.

The temperature gradients of the CNT@CNT and its component layers inner CNT, outer CNT and extracted from Figure 4(a) are $0.060 \mathrm{~K} / \AA$ (CNT@CNT), 0.059 K/ $\AA$ (inner layer), and $0.061 \mathrm{~K} / \AA$ (outer layer), while, as shown in Figure 4(d), the heat flows of these structures are $14.4 \mathrm{eV} / \mathrm{ps}$ (CNT@CNT), $16.2 \mathrm{eV} / \mathrm{ps}$ (inner layer), $30.6 \mathrm{eV} / \mathrm{ps}$ (outer layer), respectively. Based on these results, we can see that the temperature gradient of CNT@CNT is identical to the results of their component CNT layers. The heat flux of CNT@CNT is the sum of values of the inner and outer CNTs, which leads to the enhanced thermal conductance observed in CNT@CNT as shown in Figure 3(a). LikeCNT@CNT, the heat flux of CNT@BNNT shown in Figure 4(b) is also the sum of the results of its inner and outer nanotubes. However, the temperature gradient of CNT@BNNT is between the values of its inner CNT and outer BNNT components. This fact accounts for the slight discrepancy of the thermal conductance of CNT@BNNT from the sum of the values of its inner CNT and outer BNNT components 
as shown in Figure 3(b). Similar to CNT@CNT and CNT@BNNT, the heat flow of CNT@MSNT nearly equals to the sum of the values of its inner CNT and outer MSNT components (see Figure 4(c)) in spite of the fact that its increment is much smaller when compared to improvement in CNT@CNT and CNT@BNNT. On the other hand, different toCNT@CNT and CNT@BNNT, the temperature gradient of CNT@MSNT is greatly larger than the value of its inner CNT layer (see Figure 4(f)). The greatly increased temperature gradient of CNT@MSNT due to the coating of outer MSNT layer is thus responsible for the reduced thermal conductance of CNT@MSNT observed in Figure 3(c).
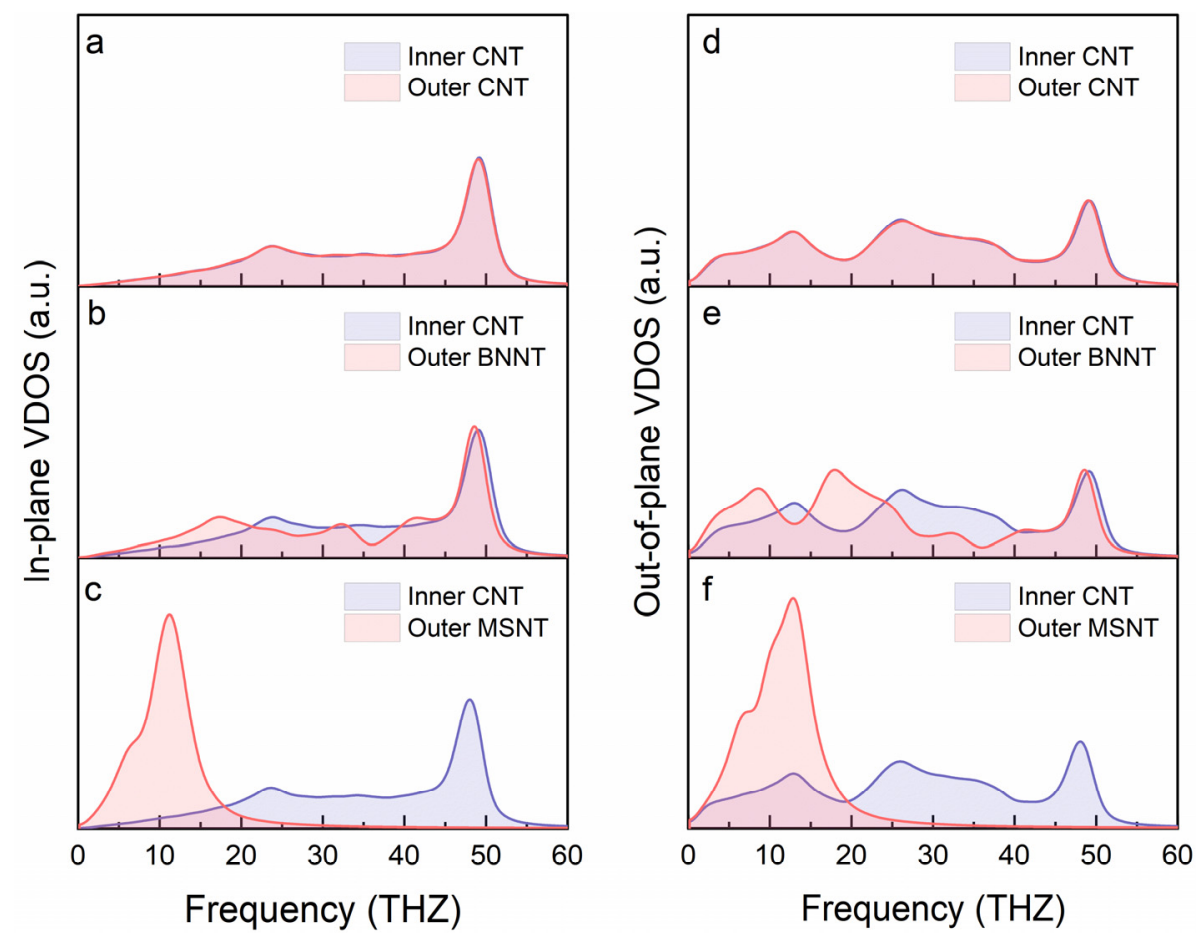

Figure 5. The in-plane (left) and out-of-plane (right) VDOS for inner and outer nanotubes of 4040CNT@4545CNT (up),4040CNT@4545BNNT (middle), and 4040CNT@3838MSNT (down).

From the above results of double-walled CNTs and two 1D vdW heterostructures, we can see that the outer nanotube coating can affect the thermal conductance of 1D $\mathrm{vdW}$ heterostructures through changing the heat flux and the temperature gradient. Specifically, the coating nanotube can provide an additional heat conducting path that can enhance the thermal conductance, while the temperature gradient may increase due to the coating of outer nanotube, which may reduce the thermal conductance. The competition between these two factors results in difficult effects of different coating 
nanotube materials on the thermal conductance of $1 \mathrm{D}$ vdW heterostructures. For example, in CNT@CNT and CNT@BNNT, the change in the thermal conductance is predominated by the enhancement in heat flux is to that in the temperature gradient, while the enhancement temperature gradient turns to be the dominant factor in CNT@MSNT.

The VDOS of inner and outer nanotubes in double-walled CNTs and two 1D vdW heterostructures is compared in Figure 5 to further provide a microscopic insight into the enhancement/hindering effects of different coating nanotubes on the thermal conductance of 1D vdW heterostructures. The in-plane VDOS is based on the atomic velocity along the $z$ direction, i.e., the axial direction, while the out-of-plane VDOS is based on the atomic velocity along other two directions. It is found that as for both VDOS, the overlap area between inner CNT and outer nanotube layer follows the order CNT@CNT>CNT@BNNT>CNT@MSNT, which indicates no, weak and strong interfacial phonon scattering in them, respectively. The diversity of phonon overlaps of these nanotubes are attributed to their different lattice structures. For example, BNNT has a lattice structure extremely analogue to that of CNT, even though the relative atomic mass of boron and nitrogen is slightly different from that of carbon. As a result, BNNT and CNT almost have the same distribution of VDOS. However, when compared to BNNT and CNT, MSNT has a more complicated atomic structure, in which each molybdenum atom is surrounded by six sulphur atoms. In other words, a molybdenum atom layer in MSNT is sandwiched by two sulphur atom layers. ${ }^{41}$ Moreover, the relative atomic mass of molybdenum is greatly larger than that of carbon. These differences lead to a VDOS of MSNT significantly different to that of CNT. Specifically, as shown in Figure 5(c) and (f), the frequency of VDOS of MSNT is much smaller than that of its CNT counterpart. 

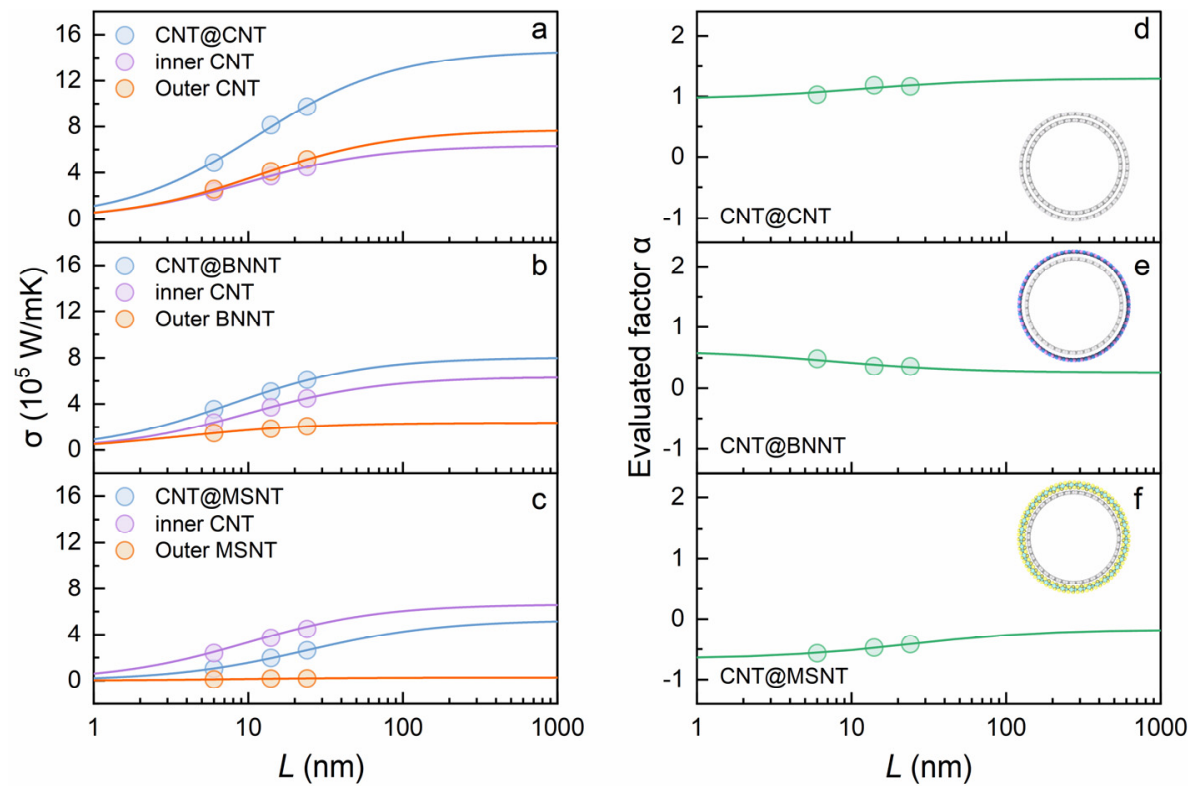

Figure 6. Length-dependent thermal conductance $\sigma$ (left) and evaluated factor $\alpha$ (right) of 4040CNT@4545CNT (up),4040CNT@4545BNNT (middle), and 4040CNT@3838MSNT (down). The circle and solid line are results obtained from NEMD simulation and Eq (4), respectively.

In Figure 6, we show the thermal conductance of CNT@CNT, CNT@BNNT, and CNT@MSNT with different effective lengths in the heat flux direction. The results of their component layer are also shown here. The effective length denotes the linear region of temperature distribution as plotted in Figure 2(a) instead of the total length of system. Thus, the nanotubes with a length ranging from $10 \mathrm{~nm}$ to $30 \mathrm{~nm}$ have an effective length ranging from $6 \mathrm{~nm}$ to $24 \mathrm{~nm}$. According to the kinetic theory, the phonons transport of low-dimensional materials will transform from ballistic regime to diffusive regime with increasing length, which results in the increase of thermal conductivity in this process until approaching a constant value. The length-dependent thermal conductance can be expressed as: ${ }^{42,43}$

$$
\sigma=\frac{\sigma_{\infty}}{\left(\frac{l_{p}}{L}+1\right)}
$$

where $\sigma_{\infty}$ is the length-independent thermal conductance, and $l_{p}$ is the effective phonon mean free path. As plotted in Figure 6, the thermal conductance $\sigma$ of all nanotubes is graphically shown as a function of the length $L$. The thermal conductance of all nanotubes is found to increases as their $L$ grows. By fitting Eq 6 to our NEMD results shown in Figure 6, the values of $\sigma_{\infty}$ are $6.639 \times 10^{5} \mathrm{~W} / \mathrm{mK}$ and $7.729 \times 10^{5} \mathrm{~W} / \mathrm{mK}$ for $4040 \mathrm{CNT}$ and 4545CNT, respectively. Thus, the corresponding length-independent thermal conductivities $k_{\infty}$ of these two materials are, respectively, $1078.97 \mathrm{~W} / \mathrm{mK}$ and 
$1164.03 \mathrm{~W} / \mathrm{mK}$. Based on the same method, the values of $\sigma_{\infty}$ extracted for BNNT,

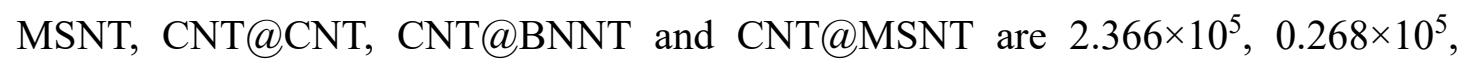
$14.620 \times 10^{5}, 8.043 \times 10^{5}$ and $5.269 \times 10^{5} \mathrm{~W} / \mathrm{mK}$, respectively. The corresponding values of $k_{\infty}$ of these materials are 172.78, 19.55, 1170.72, 644.06 and $267.89 \mathrm{~W} / \mathrm{mK}$, respectively. The value of the factor $\alpha$ of CNT@CNT, CNT@BNNT and CNT@MSNT with different lengths is shown in Figure 6 (d)-(f). Based on Eq. 6, the estimated lengthindependent values of factor $\alpha$, i.e., $\alpha_{\infty}$ of CNT@CNT, CNT@BNNT and CNT@MSNT are, respectively, 1.20, 0.21 and -0.21, which are consistent with the results obtained from structures with the length of $20 \mathrm{~nm}$. Based on the values $\alpha_{\infty}$, from which the length dependence effect is excluded, we can further conclude that both CNT and BNNT coatings can enhance the thermal conductance of $1 \mathrm{D}$ vdW heterostructures, while the MSNT coating has an opposite effect.

\subsection{Thermal transport in triple-walled nanotube heterostructure}

The strain energy of single-walled MSNT is much higher than for single-walled CNT and BNNT, due to its thickness containing three atom layers much higher than single atom thickness of the latter two. This fact leads to the fact that the CNT-BNNT are taken as base to support the MSNT and form the triple-walled heterostructure. In this section, taking CNT@BNNT@MSNT with the length of 20nm as an example (Figure 7(a)), we investigate the thermal transport in $1 \mathrm{D} v \mathrm{vW}$ heterostructures composed of three layers of nanotubes. By obtaining the temperature gradient from the linear region of temperature distribution shown in Figure 7(b) and the cumulative energy change shown in Figure 7(c), the thermal conductivity and thermal conductance are calculated as $52.873 \mathrm{~W} / \mathrm{mK}$ and $1.447 \times 10^{5} \mathrm{~W} / \mathrm{mK}$, respectively. The corresponding factor $\alpha$ is thus obtained as - 0.61 , which is larger than the value - 0.47 of CNT@MSNT. This indicates that the outer coating layers of CNT@BNNT@MSNT have a more significant reduction effect on the thermal transport property of carbon nanotube base, which is in contrast to our expectation, since it is expected that the thermal conductance of CNT@BNNT@MSNT should be smaller than that of CNT@BNNT but larger than that of CNT@MSNT. Therefore, we further calculate the heat flux and temperature distributions to understand this discrepancy. 

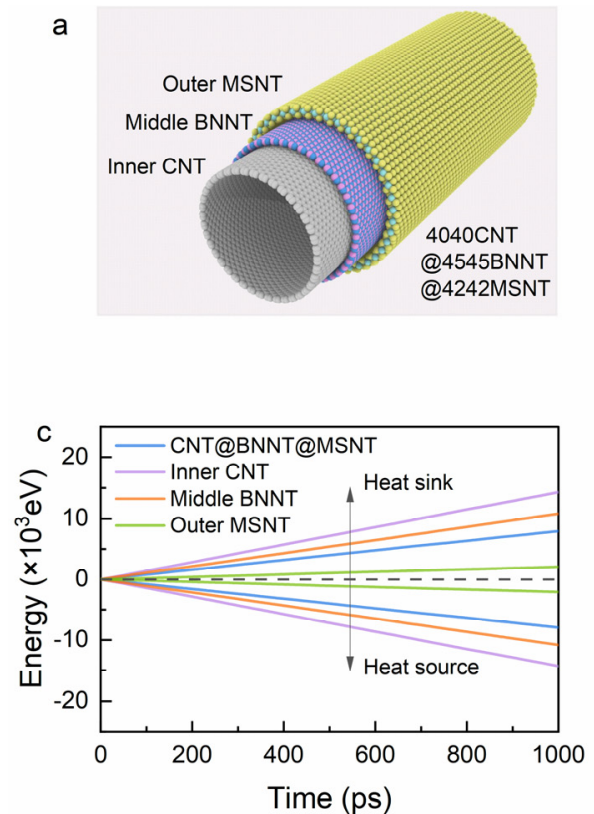
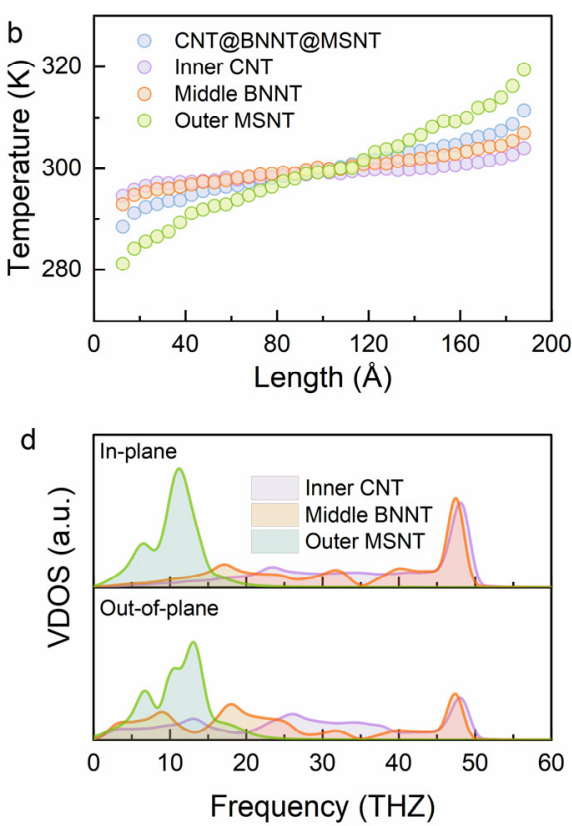

Figure 7. (a) The atomic structure, (b) temperature profile, and accumulated energy changes of CNT@BNNT@MSNT heterostructure. In plot (c), accumulated energy changes of corresponding isolated inner, middle and outer NTs are calculated to compare with the heterostructure. (d) The comparison of the in-plane (up) and out-of-plane VDOS (down) of inner CNT, middle BNNT, and outer MSNT in CNT@BNNT@MSNT.

The temperature distribution of CNT@BNNT@MSNT together with the result of its three nanotubes components is plotted in Figure 7(b). From this figure, we find that the temperature gradient of CNT@BNNT@MSNT is $0.0875 \mathrm{~K} / \AA$, which is between the values of its middle BNNT $(0.0523 \mathrm{~K} / \AA)$ and outer MSNT $(0.1633 \mathrm{~K} / \AA)$ layers, and much larger than the value of its inner CNT layer $(0.0280 \mathrm{~K} / \AA)$. Similar to the above 1D vdW heterostructures with two layers, the difference in the temperature gradient of three nanotube composites of CNT@BNNT@MSNT can be explained by their VDOS distributions in illustrated in Figure 7(d), which show the diversity of phonon overlaps owing to their different lattice structures. As plotted in Figure 7(c), the total heat flux ofCNT@BNNT@MSNT is 7.9 eV/ps, which is smaller than the values of its isolated inner CNT (14.4 eV/ps) and middle BNNT (10.8 eV/ps) layers, and much larger than the value of its isolated outer MSNT $(2.1 \mathrm{eV} / \mathrm{ps})$ layer. However, as for its counterpart with two layers, i.e., CNT@CNT, CNT@BNNT and CNT@MSNT, the heat flux is very close to the sum of the values of its inner and outer nanotubes (see Figure 4(d)(f)). Hence, the suppression of heat flux in CNT@BNNT@MSNT is responsible for its significant reduction of thermal conductivity. 


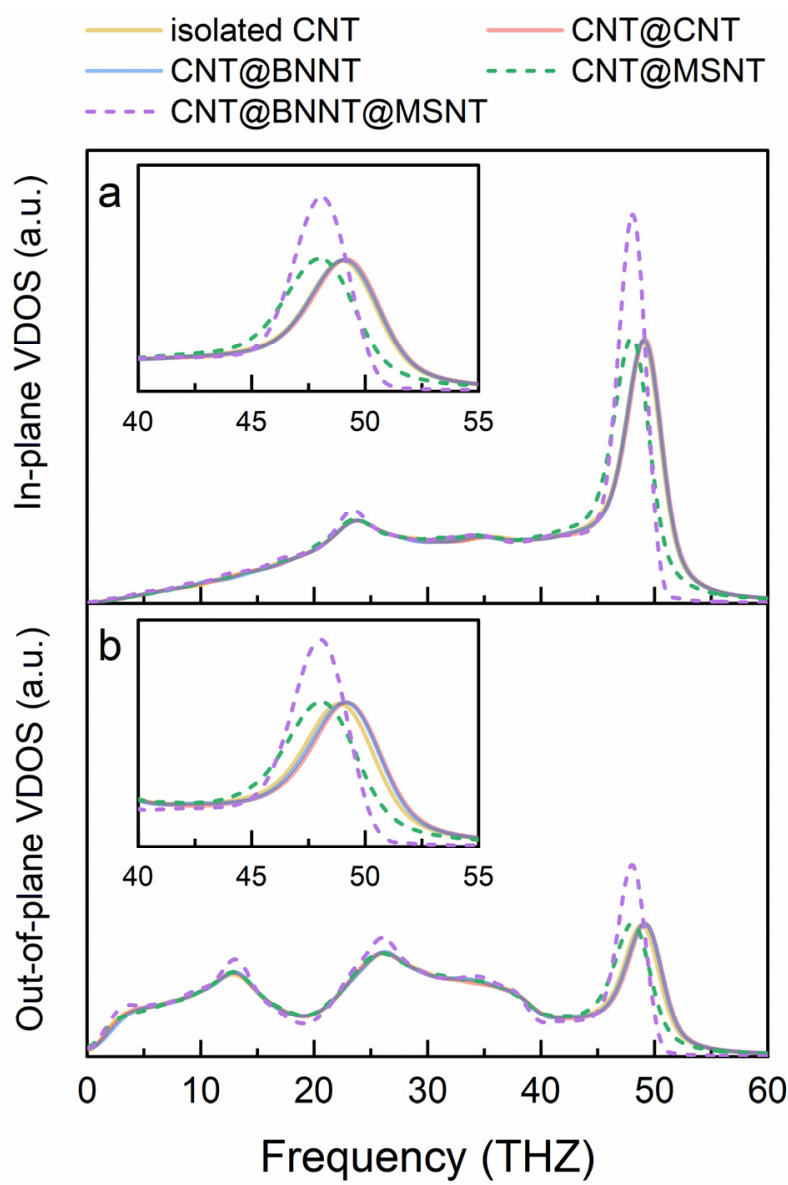

Figure 8. (a) In-plane and (b) out-of-plane of VDOS of CNT in different heterostructures including CNT@CNT,CNT@BNNT,CNT@MSNT, andCNT@BNNT@MSNT. Herein, the isolated CNT are calculated for sake of comparison. The inset zooms out the PDOS difference at frequency regime at $40 \sim 55 \mathrm{THZ}$.

In order to better understand the reduced heat flux observed in CNT@BNNT@MSNT, in Figure 8 we compare both in-plane and out-of-plane VDOS of CNT layer in different $1 \mathrm{D} \mathrm{vdW}$ heterostructures. For the sake of comparison, the value of an isolated CNT is also shown in Figure 8. It is clearly found that the VODS of innerCNT of CNT@CNT andCNT@BNNT is identical to that of the isolated CNT, whose highest peaks are all around $49 \mathrm{THz}$. However, as for inner CNT of CNT@MSNT, the peak of VDOS slightly moves leftward to $48 \mathrm{THz}$. The VDOS of innerCNT of CNT@BNNT@MSNT similarly has the highest peak around 48 THz. However, the intensity of the same phonon peaks of CNT@BNNT@MSNT is greatly increased, especially at the high frequency regime $(45 \sim 50 \mathrm{THz})$. The increased phonon peaks at high frequency regime can be attributed to the possible compression existing on the inner CNT layer, similar to a solid under pressure loading. ${ }^{44,45}$ This compression 
can be understood by the fact that in CNT@BNNT@MSNT the inner CNT is constrained by BNNT@MSNT, which thus can more significantly suppress its vibration compared with its counterpart in CNT@CNT and CNT@BNNT. This explains the origin of suppressed heat fluxion and further reduced thermal conductance inCNT@BNNT@MSNT.

\subsection{Thermal transport in the interface of double-walled nanotube heterostructures}
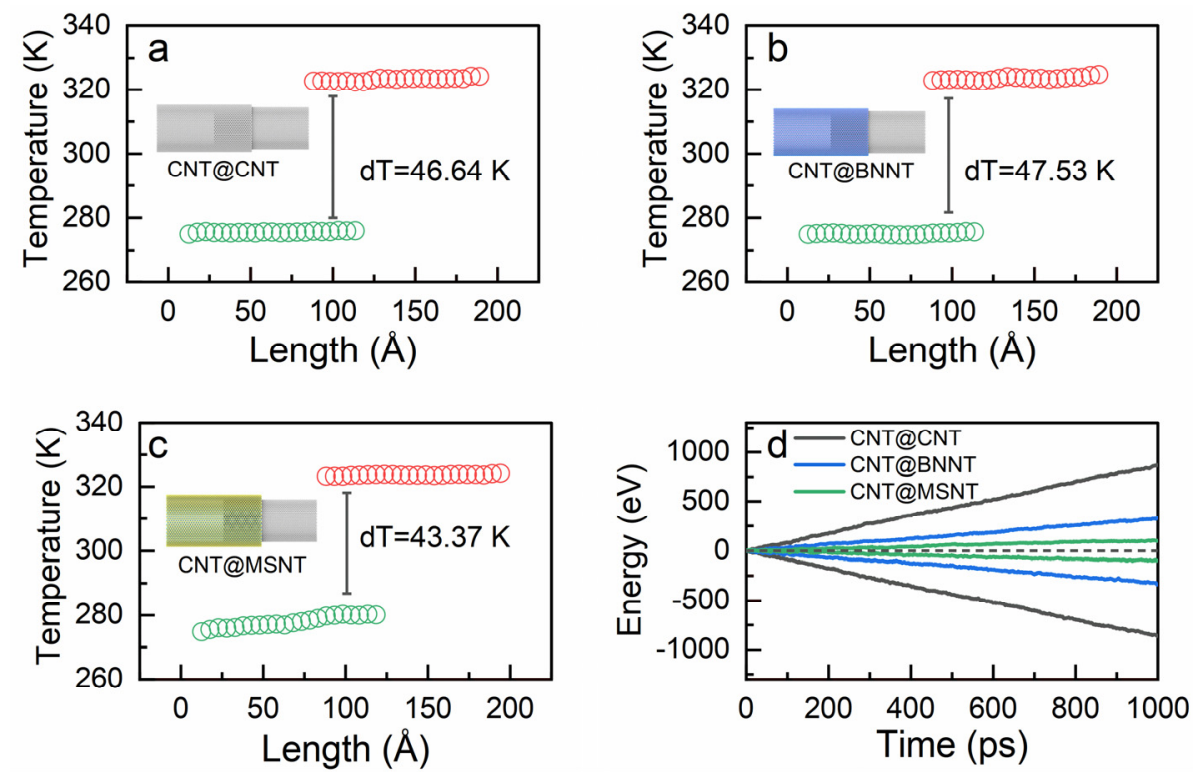

Figure 9. Temperature profiles of (a)CNT@CNT, (b) CNT@BNNT, and (c)CNT@MSNT along axial direction and (d) their corresponding accumulated energy changes in cold and hot regions during interfacial thermal conductance calculation at $300 \mathrm{~K}$.

Since the 1D van der Walls heterostructure has promising potentials in nanoscale thermal management and thermal circuits, investigating the thermal conductance at the interface between inner and outer NTs is also important for better understanding the heat dissipation mechanism. In this section, the interfacial thermal conductance of two double-walled 1D vdW heterostructures CNT@BNNT and CNT@MSNT are calculated to compare with CNT@CNT, i.e., double-walled CNT. In Figure 9 we show the interfacial temperature difference and heat flux in CNT@CNT, CNT@BNNT and CNT@MSNT. Here, all 1D vdW heterostructures are assumed to have the same overlap area. It is found that the interfacial temperature differences in CNT@CNT, CNT@BNNT and CNT@MSNT are, respectively, 46.64 K, 47.53 K, and 43.37 K, while their heat fluxes are $0.863 \mathrm{eV} / \mathrm{ps}, 0.322 \mathrm{eV} / \mathrm{ps}$ and $0.107 \mathrm{eV} / \mathrm{ps}$, respectively. From these results, we can see that there is no significant temperature gradient in the 
inner and outer nanotube layers of CNT@CNT and CNT@BNNT, since their thermal conductance along the axial direction is much larger than that along the interface direction. On the contrary, a temperature gradient is observed in the outer MSNT layer ofCNT@MSNT, owing to its relatively low in-plane thermal conductance.

According to Eq 3, values of the interfacial thermal conductance of CNT@CNT, CNT@BNNT and CNT@MSNT were calculated, which are 4.323 MW/m²K, 1.584 $\mathrm{MW} / \mathrm{m}^{2} \mathrm{~K}$, and $0.578 \mathrm{MW} / \mathrm{m}^{2} \mathrm{~K}$, respectively. The interfacial thermal conductance of CNT@CNT is about three times larger than that of CNT@BNNT and is about seven times larger than that of CNT@MSNT. The different values of the interfacial thermal conductance observed in different structures can be explained by different phonon coupling modes as detailed above, in which the VDOS of CNT@CNT, CNT@BNNT and CNT@MSNT respectively shows perfect, highly and scantly overlapped. In contrast to their high thermal conductivity along axial direction, the weak interface heat transport ability can be a drawback of $1 \mathrm{D}$ vdW heterostructures in the nanoelectronics applications, since they are hard to dissipate heat through their interfaces to surroundings.

\section{Conclusions}

In this paper, the in-plane and interfacial thermal transport behaviors in 1D heterostructures are investigated by using NEMD simulations. As for the in-plane thermal conductance of the 1D heterostructures containing two component layers, i.e., (CNT@BNNT and CNT@MSNT), it is found that the BNNT coating layer in CNT@BNNT can increase the thermal conductance of the CNT base by 36\%, which is similar to the enhancement effect observed in double-walled CNT, i.e., CNT@CNT though a CNT coating can increase the thermal conductance of CNT base by $134 \%$. Different toCNT@CNT and CNT@BNNT, the MSNT coating layer in CNT@MSNT has an opposite effect, which can reduce the thermal conductance of the CNT base by $47 \%$. The different enhancement/hindering effects of different coating layers on the thermal conductance of different $1 \mathrm{D} \mathrm{vdW}$ heterostructures is attributed to the competition between heat flux and temperature gradient of inner CNT base and outer nanotube coatings, since inner CNT and outer nanotube may have different lattice structures and relative atomic masses, which thus can result in varied VDOS overlapping degree in different $1 \mathrm{D}$ vdW heterostructures. 
In addition to $1 \mathrm{D} \mathrm{vdW}$ heterostructures containing two layers, by taking CNT@BNNT@MSNT as an example, we also investigate the thermal transport behaviors of their counterparts containing three layers. An unexpected reduction of $61 \%$ is observed in the thermal conductance of this $1 \mathrm{D} v \mathrm{vdW}$ heterostructure, which is even larger than the reduction of $47 \%$ observed in the thermal conductance of CNT@MSNT. Such significant reduction of thermal conductance observed in CNT@BNNT@MSNT is caused by the suppression of heat flux owing to vibration suppression by two outer nanotube layers. The interfacial thermal conductance of CNT@CNT, CNT@BNNT and CNT@MSNT were also examined to provide quantitative understanding. We found that the interfacial thermal conductance of CNT@CNT is 2.7 times of CNT@BNNT, and 7.5 times of CNT@MSNT. The relative difference of their thermal conductance could be explained by the phonon coupling: the VDOS of inner and outer NTs in CNT@CNT, CNT@BNNT, and CNT@MSNT are perfectly overlapped, highly overlapped, and scantly overlapped, respectively.

\section{Conflicts of interest}

There are no conflicts to declare.

\section{Acknowledgements}

This study was supported by the National Key R\&D Program of China (no. 2018YFB1502600) and the National Natural Science Foundation of China (nos. 11932005, 11772106, and 11602074). 


\section{References}

1. Novoselov, K. S.; Mishchenko, A.; Carvalho, A.; Castro Neto, A. H., 2D materials and van der Waals heterostructures. Science 2016, 353 (6298), aac9439.

2. Withers, F.; Del Pozo-Zamudio, O.; Mishchenko, A.; Rooney, A. P.; Gholinia, A.; Watanabe, K.; Taniguchi, T.; Haigh, S. J.; Geim, A. K.; Tartakovskii, A. I.; Novoselov, K. S., Light-emitting diodes by band-structure engineering in van der Waals heterostructures. Nature Materials 2015, 14 (3), 301-306.

3. Hong, X.; Kim, J.; Shi, S.-F.; Zhang, Y.; Jin, C.; Sun, Y.; Tongay, S.; Wu, J.; Zhang, Y.; Wang, F., Ultrafast charge transfer in atomically thin MoS2/WS2 heterostructures. Nature Nanotechnology 2014, 9 (9), 682-686.

4. Georgiou, T.; Jalil, R.; Belle, B. D.; Britnell, L.; Gorbachev, R. V.; Morozov, S. V.; Kim, Y.-J.; Gholinia, A.; Haigh, S. J.; Makarovsky, O.; Eaves, L.; Ponomarenko, L. A.; Geim, A. K.; Novoselov, K. S.; Mishchenko, A., Vertical field-effect transistor based on grapheneWS2 heterostructures for flexible and transparent electronics. Nature Nanotechnology 2013, 8 (2), 100-103.

5. Yury Gogotsi, B. I. Y., Nested hybrid nanotubes. Science 2020, 367 (6477), 506-507.

6. Rong Xiang, e. a., One-dimensional van der Waals heterostructures. Science 2020, 367, $537-$ 542.

7. Burdanova, M. G.; Kashtiban, R. J.; Zheng, Y.; Xiang, R.; Chiashi, S.; Woolley, J. M.; Staniforth, M.; Sakamoto-Rablah, E.; Xie, X.; Broome, M.; Sloan, J.; Anisimov, A.; Kauppinen, E. I.; Maruyama, S.; Lloyd-Hughes, J., Ultrafast Optoelectronic Processes in 1D Radial van der Waals Heterostructures: Carbon, Boron Nitride, and MoS2 Nanotubes with Coexisting Excitons and Highly Mobile Charges. Nano Letters 2020.

8. Kaur, S.; Raravikar, N.; Helms, B. A.; Prasher, R.; Ogletree, D. F., Enhanced thermal transport at covalently functionalized carbon nanotube array interfaces. Nature Communications 2014, 5 (1).

9. Yao, Z.; Wang, J.-S.; Li, B.; Liu, G.-R., Thermal conduction of carbon nanotubes using molecular dynamics. Physical Review B 2005, 71 (8).

10. Salaway, R. N.; Zhigilei, L. V., Molecular dynamics simulations of thermal conductivity of carbon nanotubes: Resolving the effects of computational parameters. International Journal of Heat and Mass Transfer 2014, 70, 954-964.

11. He, T.; Li, T.; Huang, Z.; Tang, Z.; Guan, X., Mechanical and thermal properties of the coaxial carbon nanotube@boron nitride nanotube composite. Physica E: Low-dimensional Systems and Nanostructures 2019, 107, 182-186.

12. Li, S.; Qi, W.; Xiong, S.; Yu, D., Thermal conductivity of single-wall MoS2 nanotubes. Applied Physics A 2018, 124 (3).

13. Meng, H.; Ma, D.; Yu, X.; Zhang, L.; Sun, Z.; Yang, N., Thermal conductivity of molybdenum disulfide nanotube from molecular dynamics simulations. International Journal of Heat and Mass Transfer 2019, 145.

14. Jing, L.; Samani, M. K.; Liu, B.; Li, H.; Tay, R. Y.; Tsang, S. H.; Cometto, O.; Nylander, A.; Liu, J.; Teo, E. H. T.; Tok, A. I. Y., Thermal Conductivity Enhancement of Coaxial 
Carbon@Boron Nitride Nanotube Arrays. ACS Applied Materials Interfaces 2017, 9 (17), 1455514560.

15. Ding, Z.; Pei, Q.-X.; Jiang, J.-W.; Huang, W.; Zhang, Y.-W. J. C., Interfacial thermal conductance in graphene/MoS2 heterostructures. Carbon 2016, 100 (96), 888-896.

16. Ren, K.; Liu, X.; Chen, S.; Cheng, Y.; Tang, W.; Zhang, G., Remarkable Reduction of Interfacial Thermal Resistance in Nanophononic Heterostructures. Advanced Functional Materials 2020.

17. Xu, Z.; Buehler, M. J., Heat dissipation at a graphene-substrate interface. Journal of Physics: Condensed Matter 2012, 24 (47), 475305.

18. Jiang, J.-W.; Park, H. S., Mechanical properties of MoS2/graphene heterostructures. Applied Physics Letters 2014, 105 (3).

19. Srinivasan, S.; Balasubramanian, G., Reduced Thermal Transport in the Graphene/MoS2/Graphene Heterostructure: A Comparison with Freestanding Monolayers. Langmuir 2018, 34 (10), 3326-3335.

20. Ma, Y.; Dai, Y.; Guo, M.; Niu, C.; Huang, B., Graphene adhesion on MoS2 monolayer: An ab initio study. Nanoscale 2011, 3 (9), 3883-3887.

21. Haastrup, S.; Latini, S.; Bolotin, K.; Thygesen, K. S., Stark shift and electric-field-induced dissociation of excitons in monolayerMoS2andhBN/MoS2heterostructures. Physical Review B 2016, 94 (4).

22. Miwa, R. H.; Scopel, W. L., Lithium incorporation at the MoS2/graphene interface: an ab initio investigation. Journal of Physics: Condensed Matter 2013, 25 (44), 445301.

23. Plimpton, S., Fast Parallel Algorithms for Short-Range Molecular Dynamic. Journal of Computational Physics 1995, 117(1):1-19.

24. Schneider, T.; Stoll, E., Molecular-dynamics study of a three-dimensional one-component model for distortive phase transitions. Physical Review B 1978, 17 (3), 1302.

25. Lindsay, L.; Broido, D., Optimized Tersoff and Brenner empirical potential parameters for lattice dynamics and phonon thermal transport in carbon nanotubes and graphene. Physical Review $B$ 2010, 81 (20), 205441.

26. Kınacı, A.; Haskins, J. B.; Sevik, C.; Çağın, T., Thermal conductivity of BN-C nanostructures. Physical Review B 2012, 86 (11).

27. Xu, K.; Gabourie, A. J.; Hashemi, A.; Fan, Z.; Wei, N.; Farimani, A. B.; Komsa, H.-P.; Krasheninnikov, A. V.; Pop, E.; Ala-Nissila, T., Thermal transport in MoS2 from molecular dynamics using different empirical potentials. Physical Review B 2019, 99 (5).

28. Liang, T.; Phillpot, S. R.; Sinnott, S. B., Parametrization of a reactive many-body potential for Mo-S systems. Physical Review B 2009, 79 (24).

29. Stewart, J. A.; Spearot, D. E., Atomistic simulations of nanoindentation on the basal plane of crystalline molybdenum disulfide (MoS2). Modelling and Simulation in Materials Science and Engineering 2013, 21 (4).

30. Jiang, J.-W.; Park, H. S.; Rabczuk, T., Molecular dynamics simulations of single-layer molybdenum disulphide (MoS2): Stillinger-Weber parametrization, mechanical properties, and thermal conductivity. Journal of Applied Physics 2013, 114 (6). 
31. Kandemir, A.; Yapicioglu, H.; Kinaci, A.; Çağın, T.; Sevik, C., Thermal transport properties of MoS2 and MoSe2 monolayers. Nanotechnology 2016, 27 (5), 055703.

32. Girifalco, L. A.; Hodak, M.; Lee, R. S., Carbon nanotubes, buckyballs, ropes, and a universal graphitic potential. Physical Review B 2000, 62 (19), 13104-13110.

33. Mayo, S. L.; Olafson, B. D.; Goddard, W. A., DREIDING: a generic force field for molecular simulations. Journal of Physical chemistry 1990, 94 (26), 8897-8909.

34. Liang, T.; Phillpot, S. R.; Sinnott, S. B., Erratum: Parametrization of a reactive many-body potential for Mo--S systems [Phys. Rev. B79, 245110 (2009)]. Physical Review B 2012, 85 (19).

35. Ma, Y.; Dai, Y.; Guo, M.; Niu, C.; Huang, B., Graphene adhesion on $\operatorname{MoS}(2)$ monolayer: an ab initio study. Nanoscale 2011, 3 (9), 3883-7.

36. Dickey, J. M.; Paskin, A., Computer Simulation of the Lattice Dynamics of Solids. Physical Review 1969, 188 (3), 1407-1418.

37. Haile, J.; Johnston, I.; Mallinckrodt, A. J.; McKay, S., Molecular dynamics simulation: elementary methods. Computers in Physics 1993, 7 (6), 625-625.

38. Shi, J.-X.; Natsuki, T.; Lei, X.-W.; Ni, Q.-Q., Equivalent Young's modulus and thickness of graphene sheets for the continuum mechanical models. Applied Physics Letters 2014, 104 (22), 223101.

39. Huang, Y.; Wu, J.; Hwang, K. C., Thickness of graphene and single-wall carbon nanotubes. Physical Review B 2006, 74 (24).

40. Vodenitcharova, T.; Zhang, L. C., Effective wall thickness of a single-walled carbon nanotube. Physical Review B 2003, 68 (16).

41. Penghua Ying, J. Z. a. Z. Z., Mechanical properties of monolayer ternary transitional metal dichalogenides MoS2xTe2(1 - x): A molecular dynamics study. Journal of Applied Physics 2019, $126(21), 215105$.

42. Felix, I. M.; Pereira, L. F. C., Thermal Conductivity of Graphene-hBN Superlattice Ribbons. Scientific Reports 2018, 8 (1), 2737.

43. Schelling, P. K.; Phillpot, S. R.; Keblinski, P., Comparison of atomic-level simulation methods for computing thermal conductivity. Physical Review B 2002, 65 (14).

44. Wei, Z.; Yang, F.; Bi, K.; Yang, J.; Chen, Y., Thermal transport properties of all-sp2 threedimensional graphene: Anisotropy, size and pressure effects. Carbon 2017, 113, 212-218.

45. Ying, P.; Zhang, J.; Zhang, X.; Zhong, Z., Impacts of the Functional Group Substitution and Pressure on the Thermal Conductivity of ZIF-8. The Journal of Physical Chemistry C 2020, 124 (11), 6274-6283. 